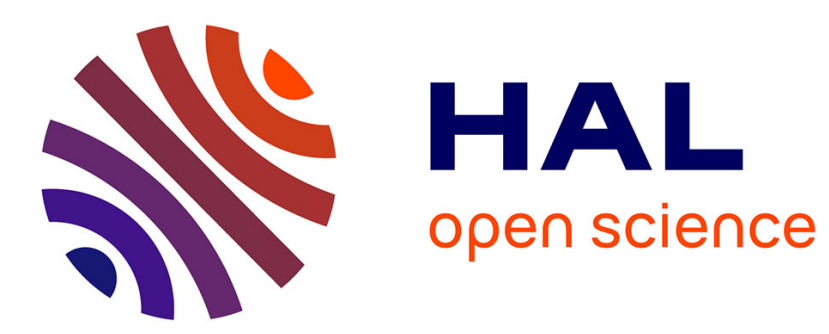

\title{
Landes-fronts 84: bilan d'une expérience d'étude des systèmes frontaux convectifs
}

J;-P. Chalon, J. L. Brenguier, J.-P. Cammas, S. Chauzy, M. Chong, M. Crochet, G. Donnadieu, Vincent Ducrocq, J.-F Gayet, P. Gondot, et al.

\section{- To cite this version:}

J;-P. Chalon, J. L. Brenguier, J.-P. Cammas, S. Chauzy, M. Chong, et al.. Landes-fronts 84: bilan d'une expérience d'étude des systèmes frontaux convectifs. La Météorologie, 1995, 10.4267/2042/51938 . hal-02000478

\section{HAL Id: hal-02000478 \\ https://hal.uca.fr/hal-02000478}

Submitted on 9 Apr 2021

HAL is a multi-disciplinary open access archive for the deposit and dissemination of scientific research documents, whether they are published or not. The documents may come from teaching and research institutions in France or abroad, or from public or private research centers.
L'archive ouverte pluridisciplinaire HAL, est destinée au dépôt et à la diffusion de documents scientifiques de niveau recherche, publiés ou non, émanant des établissements d'enseignement et de recherche français ou étrangers, des laboratoires publics ou privés. 


\section{LANDES-FRONTS 84 : BILAN D'UNE EXPÉRIENCE D'ÉTUDE DES SYSTÈMES FRONTAUX CONVECTIFS}

J.-P. Chalon (1), J.-L. Brenguier (1), J.-P. Cammas ${ }^{(2)}$, S. Chauzy ${ }^{(3)}$, M. Chong ${ }^{(1)}, M$. Crochet ${ }^{(4)}$, G. Donnadieu ${ }^{(2)}$, V. Ducrocq ${ }^{(1)}$, J.-F. Gayet ${ }^{(5)}$, P. Gondot ${ }^{(6)}$, H. Isaka ${ }^{(5)}$, P. Laroche ${ }^{(6)}$,

Y. Lemaître ${ }^{(7)}$, F.M. Ralph ${ }^{(8)}$ et S. Soula ${ }^{(3)}$

(1) Météo-France, Centre national de recherches météorologiques 42, avenue Gustave-Coriolis, 31057 Toulouse Cedex

(2) Observatoire de physique du globe de Clermont-Ferrand, Clermont-Ferrand

(3) Laboratoire d'aérologie, Université Paul Sabatier, Toulouse

(4) Laboratoire de sondages électromagnétiques de l'environnement terrestre, Toulon

(5) Laboratoire associé de météorologie physique, Clermont-Ferrand

(6) Office national de recherches aérospatiales, Meudon

(7) Centre de recherches en physique de l'environnement, Issy-les-Moulineaux

(8) Departement of Atmospheric Sciences, University of California Los Angeles, Los Angeles, Californie, USA

Expérience coopérative à laquelle ont participé de nombreux laboratoires, Landes-Fronts 84 s'est déroulée du 3 mai au 7 juillet 1984 dans les Landes et le Gers. Cette expérience a permis d'obtenir de nombreux renseignements sur les caractéristiques dynamiques, microphysiques et électriques des systèmes précipitants qui affectent le Sud-Ouest de la France. On présente ici une sélection des résultats obtenus dans les différents laboratoires ayant participé à l'expérience.

Many laboratories collaborated in the Landes-Fronts 84 campaign of observations made from 3 May to 7 July 1984 in the southwest of France. The experiment allowed much data to be gathered on the dynamics, microphysics and electrification of precipitating systems that affected the Landes and Gers. This paper gives a selection of the results obtained by the laboratories that participated.

\section{PRÉSENTATION GÉNÉRALE}

L'expérience Landes-Fronts 84 (Chalon, 1987) s'est déroulée du 3 mai au 7 juillet 1984, dans une région proche d'Aire-sur-l'Adour, s'étendant sur les Landes et le Gers. Elle était motivée par l'opportunité d'acquérir des jeux de données pouvant permettre :

- D'améliorer la prévision d'occurrence et d'intensité de certains systèmes convectifs qui affectent le Sud-Ouest, ainsi que l'estimation des précipitations qui les accompagnent.

- D'étudier les effets des phénomènes convectifs de petite et moyenne échelle sur la structure et l'évolution des systèmes frontaux, mais aussi d'en améliorer la paramétrisation dans les modèles de prévision synoptique. 
- D'étudier les mécanismes de formation de la foudre et les conséquences de cette dernière sur les équipements électroniques, tant à bord des aéronefs que dans les installations au sol.

Cette expérience s'inscrivait naturellement dans la continuité des programmes de recherche menés depuis plusieurs années par les laboratoires français sur l'étude des systèmes frontaux (Fronts 77), de la convection développée (Landes 79, Copt 79 et Copt 81) et des orages (Trip 82 et Trip 83). Les expériences Fronts 77 et Landes-Fronts 84 concernaient les phénomènes de petite et moyenne échelle, aspects des systèmes frontaux qui, à notre avis, demeuraient les plus mal connus en dépit d'assez nombreuses expériences antérieures. Par petite et moyenne échelle, on se réfère à la gamme d'échelles qui va de quelques kilomètres (échelle convective) à quelques centaines de kilomètres (échelle des bandes de précipitation). L'expérience Fronts 77 a permis de décrire la circulation de petite échelle au sein de systèmes frontaux d'hiver (Chalon et Gilet, 1980 ; Testud et al., 1980) ; Landes-Fronts 84 a permis de mettre en évidence l'importance des structures convectives et le caractère orageux de systèmes frontaux qui se développent au printemps ou en été. Les acquis scientifiques, technologiques et logistiques de cette expérience ont



Figure 1 - Site et dispositif expérimental de l'expérience Landes-Fronts 84. Les carrés dessinés en pointillés de part et d'autre des radars Ronsard (R1 et R2) représentent les zones de restitution des champs tridimensionnels de vent mesurables à partir de ces radars Doppler (adapté de Lemaître et al., 1989). aussi été mis à profit pour organiser l'expé-rience Fronts 87, en 1987-1988, au-dessus de la Manche, et l'expérience Pyrex, en 1990, dans une région englobant le site de Landes-Fronts 84 et le massif des Pyrénées.

Les organismes participant à Landes-Fronts 84 étaient : le Centre national d'études des télécommunications (CNET), le Centre de recherches en physique de l'environnement (CRPE/CNET), l'Établissement d'études et de recherches météorologiques ${ }^{(1)}(\mathrm{EERM})$, l'Institut et observatoire de physique du globe du Puy-deDôme (IOPG), le Laboratoire d'aérologie (LA), le Laboratoire associé de météorologie physique (LaMP), le Laboratoire de météorologie dynamique (LMD/CNRS), le Laboratoire de sondages électromagnétiques de l'environnement terrestre (LSEET), l'Office national de recherches aérospatiales (ONERA), le département de physique de la faculté des sciences d'Abidjan (Côte-d'Ivoire), le Centre d'études aéronautiques de Toulouse (CEAT).

Les fronts sont le siège d'un grand nombre de phénomènes qui mettent en œuvre une large variété de processus dynamiques, thermodynamiques, microphysiques et électriques. Cette grande variété rend la compréhension des phénomènes frontaux relativement malaisée. La difficulté est décuplée par le fait que certains des processus impliquent des échelles de mouvement allant de l'échelle de la turbulence à l'échelle planétaire. Parmi ces processus, la convection joue un rôle important dans la dynamique frontale. Elle apparaît sous différentes formes et peut être organisée en lignes ou être associée à une structure dynamique ou thermodynamique préexistante (isolée dans le secteur froid de la perturbation, en ligne dans le secteur chaud, en bande large sur la surface frontale froide, en bande étroite le long de la discontinuité frontale). Dans ce contexte, l'objectif de l'expérience Landes-Fronts 84 était de mettre en œuvre un dispositif expérimental qui permette d'élucider :

- La raison des différences observées (en termes de dynamique, de thermodynamique, de microphysique et d'électrification) entre ces différents types de phénomènes convectifs.

(1) L'Établissement d'études et de recherches météorologiques est l'ancienne dénomination de l'actuel Centre national de recherches météorologiques de Météo-France. 
- L'origine de ces différentes organisations (forçage, instabilités dynamiques, ondes de gravité...).

- Le lien avec le contexte frontal et l'interaction éventuelle de ces phénomènes convectifs avec les autres entités qui constituent le système frontal (discontinuité frontale, jet de basse couche, jet d'altitude...).

Le réseau d'observation (figure 1) était doté d'une station météorologique centrale équipée pour l'interrogation à distance de stations automatiques (4M), ainsi que pour la réception des cartes météorologiques du réseau synoptique et des images des satellites Météosat et NOAA 6, 7 et 8. Il comportait aussi quatre stations de radiosondage, deux radars météorologiques de surveillance des précipitations (Bordeaux et Toulouse), un système de deux radars Doppler en bande C (Ronsard), un radar Doppler en bande K (Rabelais), un radar à diversité de polarisation en bande $\mathrm{S}$ (Anatol), un radar VHF (72 MHz) permettant la mesure continue des profils de vitesse verticale de l'air (Provence), un sodar Doppler triple, un réseau à moyenne échelle de stations météorologiques automatiques (Patac), un spectropluviomètre (Vidiaz), une station centrale de mesure des paramètres électriques et électromagnétiques, une station de radiosondage électrique, un réseau de stations de mesures automatiques du champ électrique et trois avions instrumentés (Transall, Piper Aztec et Cessna 206).

Le jeu important de données obtenues au cours de cette expérience a permis de répondre, au moins partiellement, aux questions posées et d'obtenir :

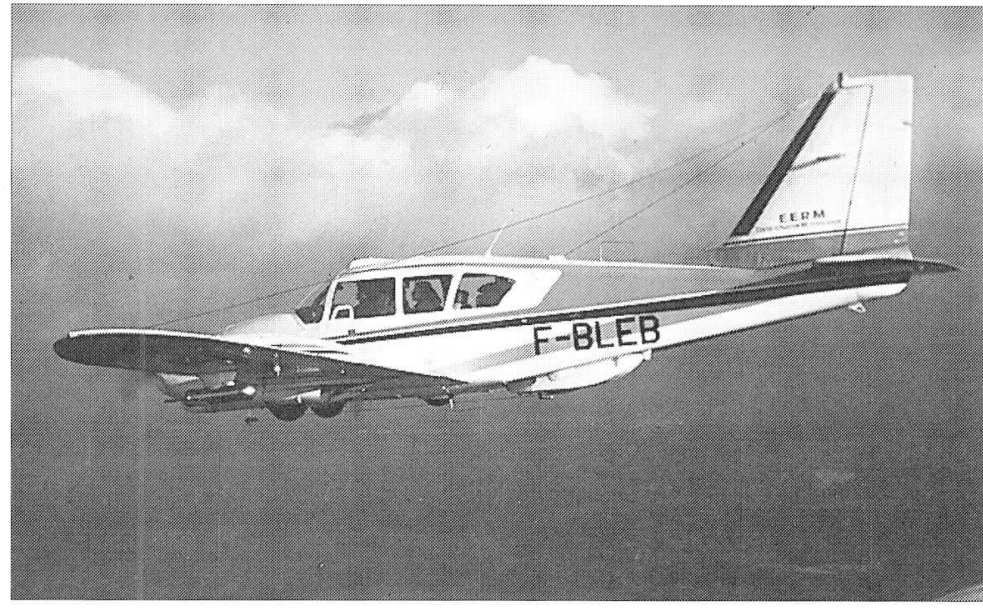

L'avion de recherche Piper Aztec de Météo-France, instrumenté pour les mesures thermodynamiques et microphysiques
- une description de la structure tridimensionnelle des systèmes convectifs observés,

- la déterminations du (ou des) mécanisme(s) de moyenne échelle à l'origine de ces systèmes,

- un diagnostic de leurs conditions d'apparition,

- une évaluation de l'importance des ondes de gravité et de leur rôle dans le déclenchement de la convection,

- une description du couplage jet d'altitude-front, - la détermination des processus microphysiques impliqués dans ces phénomènes et leur localisation dans les cellules orageuses,

- une description de la structure électrostatique des cellules orageuses,

- la mise en évidence des interactions entre les phénomènes dynamiques, microphysiques et électriques.

Ces données ont permis en particulier de révéler l'importance des processus microphysiques (condensation, évaporation, fonte) dans la dynamique à méso-échelle des phénomènes convectifs. Elles ont également confirmé l'importance d'un processus d'instabilité nouvellement découvert (l'Instabilité symétrique conditionnelle ou ISC) sur l'organisation à méso-échelle de la convection. Elles ont permis la mise en évidence de couplages entre les différentes entités constituant le système frontal, en particulier entre les fronts d'altitude et de surface, entre la convection et le jet de basse couche, entre les processus microphysiques et la dynamique frontale. Cependant, le dispositif expérimental mis en œuvre au cours de cette expérience n'a pas permis l'étude détaillée de ces couplages. L'expérience Fronts 87, tirant les leçons de Landes-Fronts 84, a mis en œuvre un dispositif plus complet permettant de mieux appréhender l'interaction entre les différentes échelles de mouvement impliquées dans ce couplage.

Ces résultats ont fait l'objet de plusieurs thèses (trois thèses d'État, sept thèses d'université et une thèse de doctorat dans une université américaine), ainsi que de nombreuses publications dans des revues scientifiques internationales. Les documents disponibles sont référencés dans la bibliographie qui est organisée en deux parties : la première détaille l'ensemble des documents mentionnés dans l'article ; la seconde fournit la liste des autres travaux effectués dans le cadre de l'expérience Landes-Fronts 84. Les pages 13 à 23 présentent quelques-uns de ces résultats, organisés selon les grands thèmes d'étude qui avaient servi de base à la préparation de l'expérience : dynamique et ondes de gravité, phénomènes microphysiques, phénomènes électriques. La situation des 2 et 3 juin 1984 a aussi 


\section{DYNAMIQUE DES BANDES PRÉCIPITANTES}

été l'opportunité d'une étude qui n'était pas prévue dans les objectifs initiaux de l'expérience : l'étude dynamique des frontogenèses d'altitude (page 16). Enfin, le paragraphe débutant page 24 est consacré aux acquis méthodologiques et logistiques.

\section{Bandes de précipitations associées à un front froid}
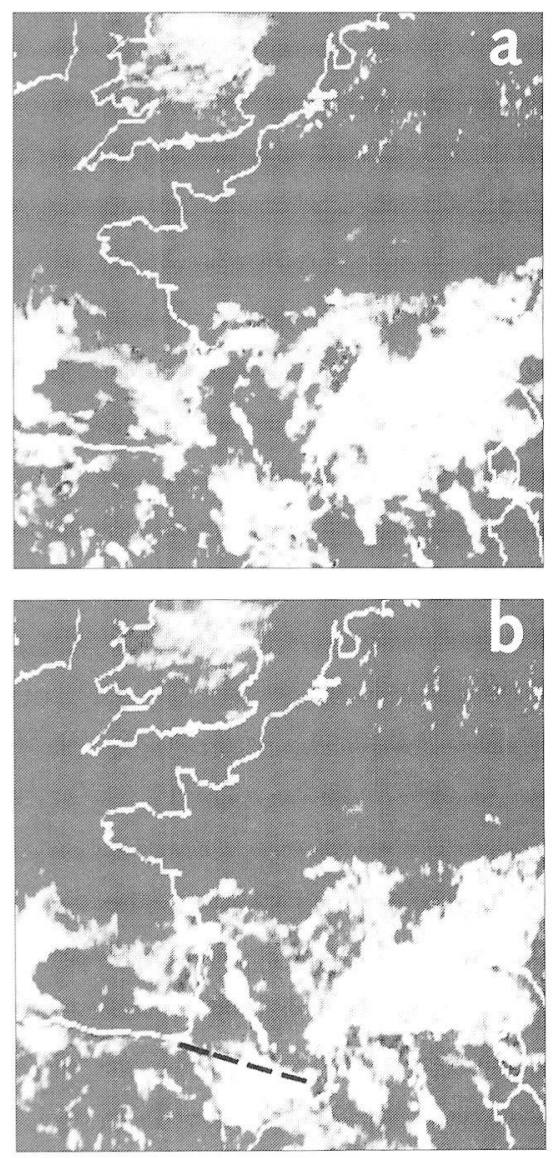

Figure 2 - Évolution temporelle de la structure nuageuse associée à la ligne de convection observée le 18 mai 1984 (a) 13 h 30 et (b) 14 h 30, et s'étendant suivant un axe Gironde-Pyrénées centrales (extrait de Lemaitre et Brovelli, 1990)
Les moyens mis en place lors de Landes-Fronts 84 ont permis d'étudier la convection organisée en lignes et en bandes, en essayant de la restituer dans son contexte synoptique pour chercher à comprendre les principales raisons de son organisation et de son déclenchement. Il s'agissait de :

- déterminer les conditions d'apparition de la convection,

- diagnostiquer le mécanisme de moyenne échelle à l'origine du déclenchement de la convection,

- décrire la dynamique des systèmes.

Les cas qui seront présentés par la suite, à titre d'illustration, ont permis de confirmer l'importance des processus de moyenne échelle dans les systèmes convectifs observés à nos latitudes, de décrire certains des mécanismes d'interaction entre les échelles de mouvement et de mettre en évidence le rôle important que jouent les processus microphysiques en phase solide (glace) dans l'intensification des circulations de moyenne échelle. Toutefois, l'interaction avec les phénomènes de grande échelle n'a pu être abordée que de façon qualitative, du fait de la présence des Pyrénées et de l'insuffisance des moyens disponibles, lors de l'expérience, pour appréhender ces échelles.

Les nuages convectifs associés à un système frontal apparaissent sous différentes formes :

- en ligne, associés à la discontinuité frontale froide au sol ou associés à une ligne de convergence induite par le relief (cas du 18 mai, du 2 juin et du 4 juin),

- en bande située dans la traîne du système frontal (cas du 4 juin),

- isolés (cas du 28 juin).

L'identification des mécanismes physiques responsables de l'organisation en bandes ou en lignes a pu être menée à bien pour plusieurs cas d'observations. Ces cas se distinguaient essentiellement par la localisation des bandes dans le système frontal (secteur chaud, discontinuité frontale, secteur froid post-frontal) et par leur lien avec les deux entités principales qui caractérisent les basses couches d'un système frontal, à savoir la discontinuité frontale froide et le jet de basse couche.

La situation du 18 mai 1984 (figure 2) a permis une description fine de l'interaction qui existe entre le jet de basse couche et la bande de précipitations étroite qui caractérise généralement la discontinuité frontale froide au sol (Lemaître et Brovelli, 1990). Ce cas, très favorable à une telle étude, présentait un forçage de grande échelle très faible et a permis d'étudier isolément les processus dynamiques liés à la présence du jet de basse couche et aux cisaillements de vent qui lui sont associés. Nous avons pu ainsi montrer que ce jet, qui résulte initialement du gradient de température dans le secteur chaud de la perturbation induite par la circulation anticyclonique, joue un rôle moteur aussi bien à moyenne échelle qu'à échelle convective dans l'entretien et l'organisation de la convection associée à la discontinuité frontale froide au sol ; la figure 3 montre la structure verticale du champ de vitesse et de réflectivité associée aux mouvements convectifs déduite des observations effectuées par les deux radars Ronsard du dispositif expérimental. Les cisaillements de vent horizontaux et verticaux produisent, en particulier, de l'instabilité symétrique ${ }^{(1)}$ qui génère une ligne étroite de convergence, dans laquelle le couplage entre instabilité de cisaillement et instabilité convective conduit à une structure périodique des mouvements convectifs dans la bande étroite de précipitations.

Le front froid secondaire observé le 4 juin 1984 a pu être décrit, au moins qualitativement, sur tout son cycle de vie (Lemaître et al., 1989). Cette perturbation, qui a donné des précipitations intenses sur la zone d'expérimentation, devait son apparition à une interaction entre la convection isolée située dans l'air froid à l'arrière d'un front froid principal et un tourbillon préexistant en altitude. Ce

(1) Instabilité résultant de l'interaction entre la force de gravité et la force de Coriolis. Cette instabilité se traduit par des mouvements ascendants inclinés (ou obliques) et non verticaux comme dans le cas de la convection orageuse (appelée également convection droite). 

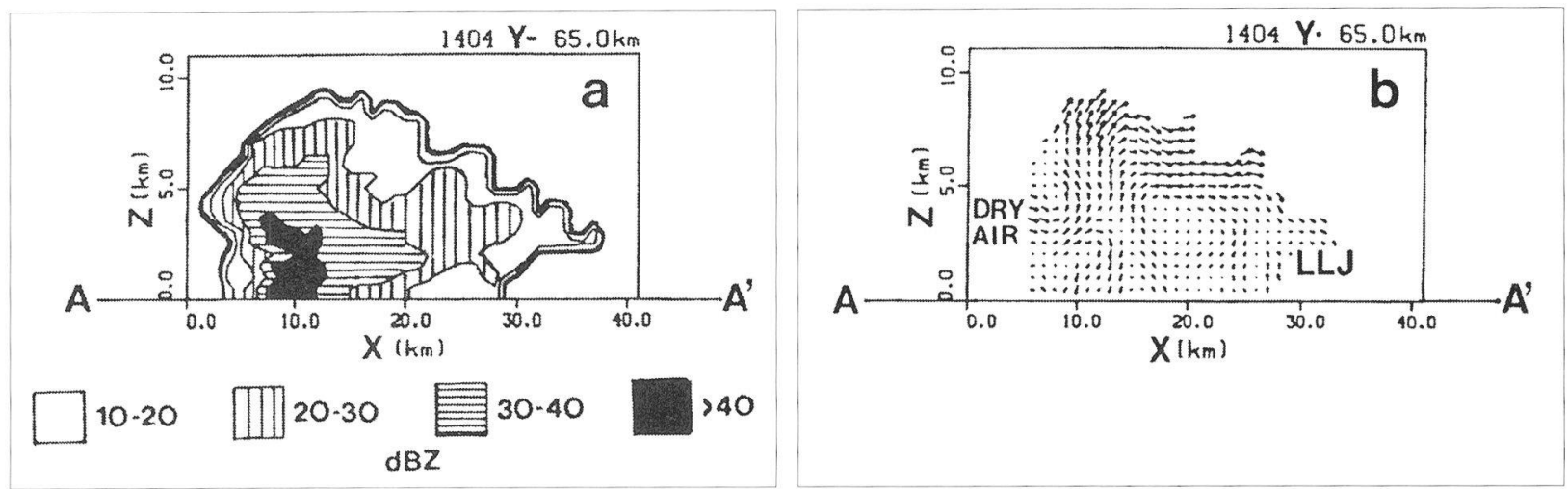

Figure 3 - Champs de réflectivité (a) et de vitesse (b) dans une coupe verticale transverse au jet de basse couche observé le 18 mai 1984. Ces champs révèlent le lien étroit entre les forts mouvements verticaux (plus de $7 \mathrm{~m} \cdot \mathrm{s}^{-1}$ ) situés au-dessus de $3 \mathrm{~km}$ d'altitude et les précipitations intenses observées. Ces mouvements verticaux résultent de l'interaction constructive entre les différents processus d'instabilité causés par la structure dynamique et thermodynamique particulière de l'atmosphère liée à la présence du jet de basse couche. Les mouvements subsidents produits par l'évaporation des précipitations intenses conduisent dans les basses couches à une convergence qui alimente la convection (extrait de Lemaitre et Brovelli, 1990).

\section{Déclenchement de bandes convectives}

phénomène a très rapidement conduit à une organisation en comma cloud ${ }^{(1)}$ de la convection, puis a évolué, sur la zone d'expérience, vers une structure en forme de «lambda» caractéristique des systèmes frontaux. Les précipitations associées au front froid avaient une organisation très proche de celle des lignes de grains tropicales. Lemaître et al. (1989) ont montré que ces précipitations résultaient de l'interaction constructive entre le forçage frontogénétique dû aux déformations induites par la circulation synoptique (qui donne l'enveloppe des précipitations), la relaxation de l'Instabilité symétrique conditionnelle (ISC) préexistant dans l'atmosphère et le soulèvement forcé de l'air chaud par la discontinuité frontale qui se comporte à plus petite échelle comme un courant de densité.

L'étude des systèmes convectifs observés le 2 juin et le 4 juin 1984 a permis de confirmer l'idée selon laquelle de nombreux systèmes convectifs observés dans nos régions ne doivent pas toujours leur présence à un forçage de grande échelle, mais peuvent, dans certains cas, résulter d'une circulation associée à un processus de moyenne échelle ; cette circulation modifie la structure verticale de l'atmosphère initialement stable (vis-à-vis des mouvements convectifs) et engendre localement des zones d'instabilité convective dans lesquelles la convection est entretenue par cette circulation (Lemaître, 1987). Ces observations montrent qu'une bonne prévision des précipitations ne peut passer que par une bonne représentation dans les modèles de ces mécanismes dynamiques de moyenne échelle.

Dans le cas du 4 juin, les différentes comparaisons faites avec les mécanismes qui peuvent théoriquement agir à moyenne échelle ont permis de montrer que l'ISC était le mécanisme mis en jeu. Il permet, en effet, d'expliquer les caractéristiques morphologiques du système convectif (vitesse de propagation, circulation en rouleaux à moyenne échelle...), mais également de rendre compte (figure 4) de la structure détaillée de cette circulation (pente des ascendances, énergétique de la circulation...) et d'expliquer les variations de pression et de température observées au passage du système. Tous ces progrès ont pu être faits grâce aux efforts consentis ces dix dernières années dans le traitement des données de radars Doppler pour obtenir, à l'échelle convective mais surtout à méso-échelle, les champs tridimensionnels de vitesse, de pression et de température nécessaires à la compréhension des processus de moyenne échelle et de leur interaction avec la convection.

Le cas de la convection organisée observée dans l'après-midi du 2 juin 1984 a été sélectionné en raison de la particularité de la situation météorologique: absence de couche conditionnellement instable et développement de convection profonde. Les conditions météorologiques associées ont fait l'objet d'une étude détaillée (Lefèvre, 1987 ; Trraff, 1988). 
Figure 4 - Coupe verticale de la circulation de moyenne échelle et du champ de réflectivité associés au système convectif post-frontal observé le 4 juin 1984. La circulation en rouleaux (notés $A$ et $B$ ) observée, d'axe parallèle au cisaillement du vent moyen dans la couche perturbée, est la signature de I'Instabilité symétrique conditionnelle (extrait de Lemaître et Scialom, 1992).

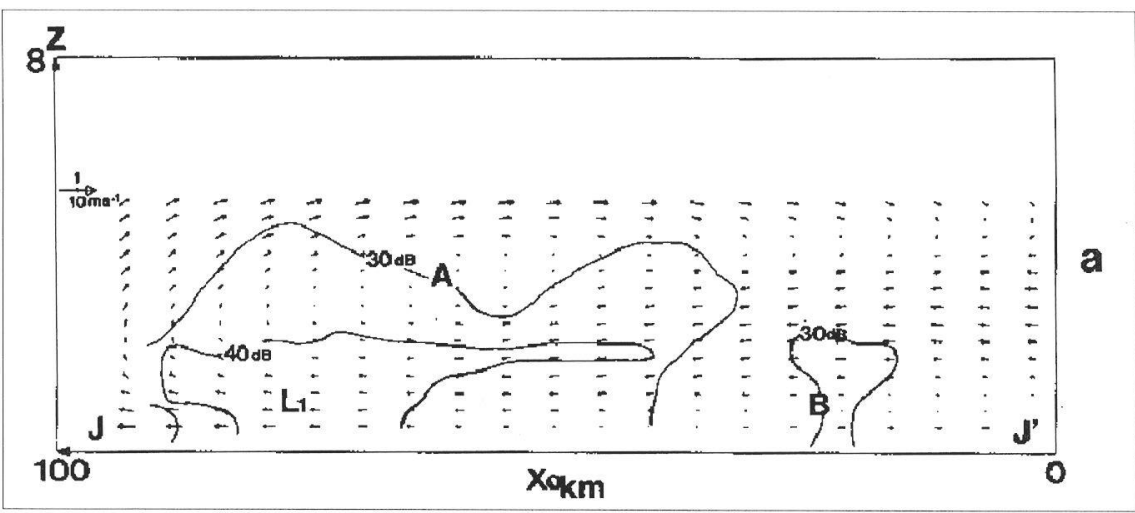

L'analyse effectuée par Trraff (1988) a montré la présence d'une zone de convergence induite par le relief pyrénéen et la possibilité de déclenchement de la convection profonde par l'ascension de particules d'air venant de la Méditerranée dans le courant transpyrénéen. Les données d'observation ne permettent pas de déterminer précisément l'origine et le mécanisme intervenant dans le développement de cette convergence ; toutefois, la déviation du vent surgéostrophique, induit dans la couche limite par le courant de descente transpyrénéen, est un des mécanismes les plus probables. Par ailleurs, la présence de ce courant de descente dans la partie occidentale des Pyrénées a été démontrée par l'analyse des données et sa vraisemblance confirmée par l'utilisation du modèle analytique de Blumen.

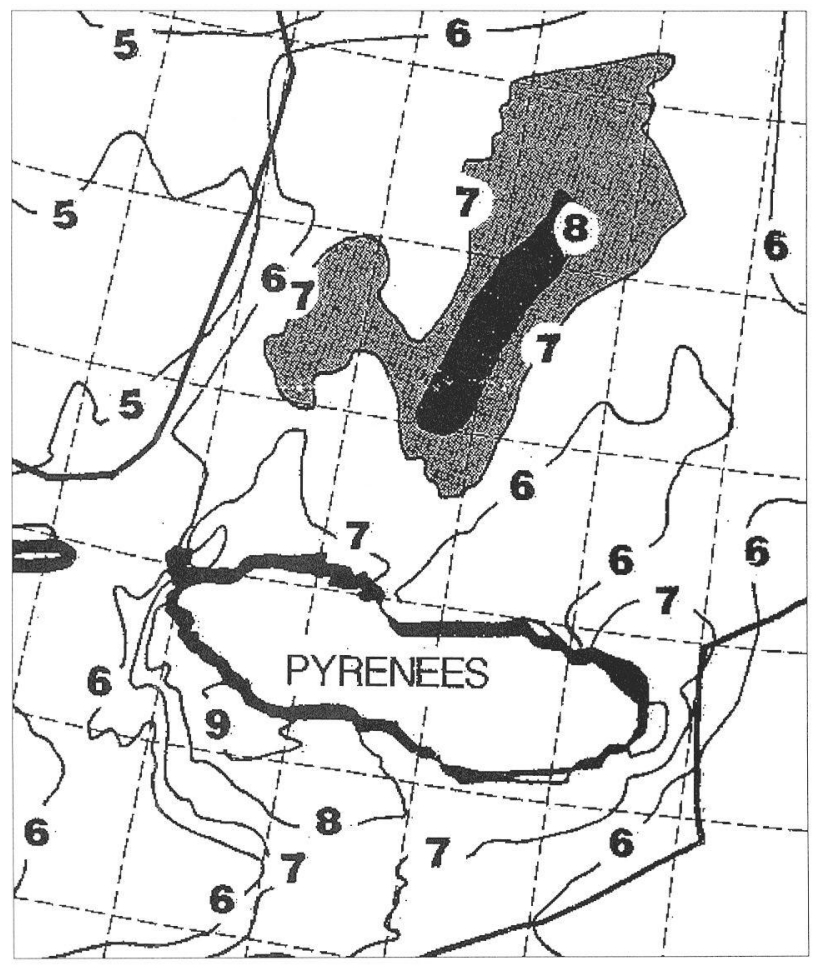

Figure 5 - Humidité spécifique (isolignes tous les $1 \mathrm{~g} \cdot \mathrm{kg}^{-1}$ ) à $850 \mathrm{hPa}$, prévue pour le 2 juin 1984 à 15 h UTC par le modèle Péridot avec une résolution quatre fois plus fine $(9,5 \mathrm{~km})$ que celle du modèle opérationnel. La région grisée correspond à la structure en bande prévue. Les contours des côtes et des reliefs sont indiqués en traits gras.
Les bandes précipitantes étudiées par Lefèvre (1987) et Ducrocq et Willemet (1988) ont fait l'objet d'une étude numérique à l'aide du modèle Péridot. Alors que le modèle opérationnel, de résolution $35 \mathrm{~km}$, donne une représentation assez éloignée de la réalité (noyaux difformes à $850 \mathrm{hPa}$ ), un modèle de maille horizontale quatre fois plus fine (résolution de $9,5 \mathrm{~km}$ ) est capable de prévoir une structure en bande des champs d'ascendance et d'humidité, avec une localisation relativement correcte dans l'espace et le temps (figure 5). Ainsi, l'évolution prévue au cours de l'après-midi sur une période de six heures présente-t-elle une certaine analogie avec ce qui a été observé, par exemple, le redressement nord-sud de l'une des bandes.

Ces résultats ont permis d'étudier le mécanisme de formation d'une des bandes précipitantes simulées par le modèle. La bande précipitante étudiée et son environnement possèdent des caractéristiques communes avec la théorie de l'instabilité symétrique. En effet :

- La bande s'est développée dans une région où le tourbillon potentiel humide était, dans son ensemble, négatif et elle est plus marquée dans les zones de plus forte instabilité.

- L'environnement à méso-échelle est approximativement bidimensionnel.

- Les circulations associées à la bande sont organisées en forme de rouleaux en pente avec une branche ascendante étroite et une subsidence selon les surfaces isentropiques.

- La bande est alignée dans la direction du cisaillement vertical du vent.

L'instabilité symétrique conditionnelle a donc pu jouer un rôle dans la formation de la bande en question. Toutefois, cette hypothèse n'a pu être confirmée par une première approche de l'énergétique des circulations simulées (Ducrocq et Willemet, 1988). Nous avons donc décidé de poursuivre ce travail par le développement d'une méthode générale d'étude de l'énergétique des circulations associées à des phénomènes bidimensionnels de méso-échelle, et par sa qualification dans un cadre plus théorique, comme celui de simulations de cas idéalisés d'instabilité symétrique (Ducrocq, 1993). 


\section{Dynamique d'une ligne de grains extratropicale}

Alors que les lignes de grains extratropicales avaient déjà fait l'objet de nombreuses études aux États-Unis, l'observation d'une ligne de grains dans le SudOuest de la France à l'aide de radars Doppler constituait une première. Une analyse des écoulements convectifs associés à la situation du 20 juin 1984 a été effectuée (Jaubert et al., 1988 ; Chong et Jaubert, 1990) en utilisant conjointement les données des radars Doppler et les données météorologiques.

Deux types d'interaction ont ainsi pu être identifiés. Le premier concerne l'effet de l'environnement proche sur la dynamique du système. La présence d'un air sec dû aux effets orographiques (effet de foehn produit par les Pyrénées dans un flux de sud - sud-ouest, un des traits fréquents dans le Sud-Ouest de la France) a une influence majeure en empêchant toute alimentation du système en air de basses couches humide et instable. Les données radar montrent clairement l'absence de convergence de basses couches au niveau des cellules convectives (figure 6); de plus, les données météorologiques indiquent que cette convergence nécessaire à l'entretien et à la propagation du système se situe bien en amont. Le second type d'interaction concerne l'impact de l'environnement de grande échelle sur la vitesse de propagation de la ligne de grains. Cette dernière est en effet associée au déplacement de la ligne de convergence de grande échelle apparue lors d'un changement du régime de sud - sud-ouest à sud-ouest. L'impact de l'environnement de grande échelle s'est traduit par une atténuation progressive de l'effet de foehn, qui a permis au système de se propager malgré un environnement peu favorable.

Cette ligne de grains a pu être comparée à une autre ligne de grains observée en Oklahoma pour mettre en évidence les points communs et les différences fondamentales qui étaient surtout liées à l'effet de fœhn produit par la chaîne pyrénéenne.
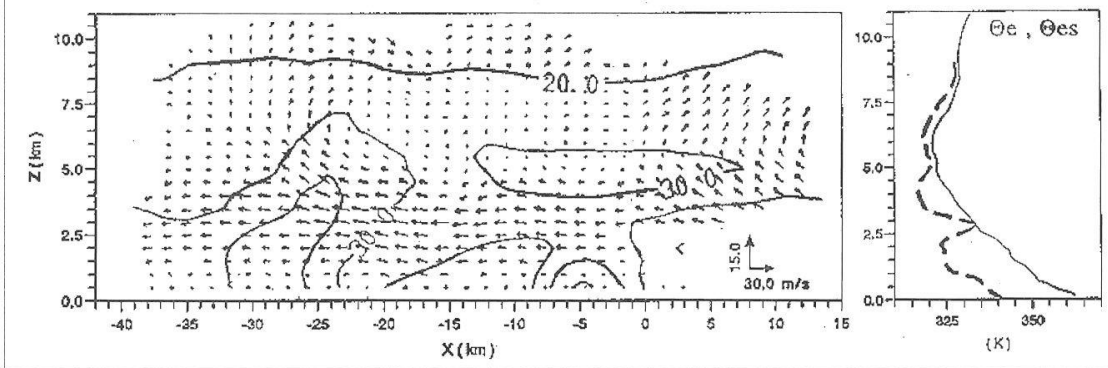

Figure 6 - Coupe verticale transverse à la ligne de grains mettant en évidence l'écoulement de I'air (flèches) associé aux zones précipitantes à 20 h 22 UTC (isocontours de réflectivité radar en $\mathrm{dBZ}$ ), et les profils verticaux de température potentielle équivalente $\theta_{\mathrm{e}}$ (trait tireté) et de température potentielle équivalente saturée $\theta_{\text {es }}$ (trait continu) à l'avant du système à $18 \mathrm{~h} 10$ UTC. Les vecteurs vent tracés ici respectent le rapport d'aspect des axes pour permettre d'appréhender les lignes de courant dans le plan considéré. L'origine des abscisses coïncide avec le début des précipitations au sol. On remarque nettement l'enclume du système et l'absence de convergence de basses couches au niveau de la cellule principale $(x=-28 \mathrm{~km})$. Les ascendances associées sont alimentées par l'air humide qui est situé autour de $3 \mathrm{~km}$ et qui surplombe une couche épaisse d'air relativement sec (adapté de Chong et Jaubert, 1990).

\section{DYNAMIQUE DES SYSTËMES JET-FRONTS}

Dans ce domaine, le premier objectif était de construire des méthodes d'analyse capables de caractériser les circulations agéostrophiques induites par la dynamique du courant-jet. La situation du $1^{\text {rr }}$ au 4 juin 1984, qui était caractérisée par une grande diversité de la circulation en altitude (intensité et courbure du courant-jet), a permis de confronter nos résultats d'analyse diagnostique aux résultats récents de modèles numériques et conceptuels (Cammas, 1988). Bien qu'aucun des objectifs scientifiques de l'expérience Landes-Fronts 84 n'ait été spécifiquement axé sur la frontogenèse d'altitude, ce travail a permis de faire débuter en France des études sur ce thème de recherche, et, déjà, de répondre à quelques questions laissées jusqu'alors sans réponse.

L'exploitation des analyses numériques des modèles de Météo-France (Péridot) et du Centre européen pour les prévisions météorologiques (CEPMMT) a montré que les circulations agéostrophiques longitudinales forcées par la courbure du flux peuvent être dominantes dans la composition du mouvement agéostrophique total, jusqu'à masquer des circulations agéostrophiques transverses, ce qui n'apparaissait pas dans les études conceptuelles et numériques les plus récentes (figure 7). Nous avons ainsi pu montrer, pour notre cas d'étude, que la circulation directe de la zone d'entrée du maximum de courant-jet, immédiatement 


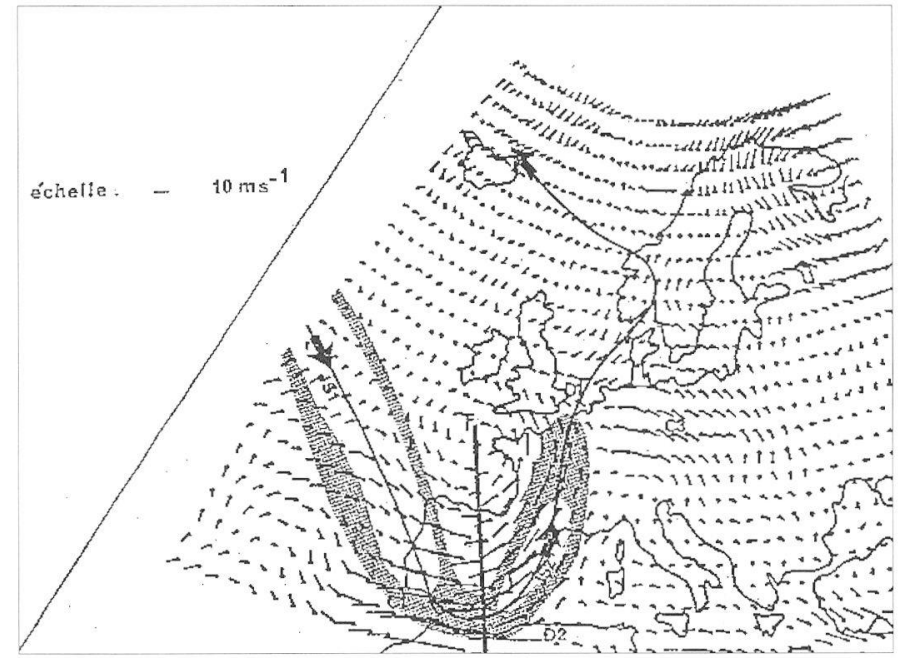

Figure 7 - Champ du vent agéostrophique total, le 3 juin 1984 à 06 h 00 UTC, sur la surface $300 \mathrm{hPa}$. Les zones en grisé correspondent aux régions où les vitesses du vent sont comprises entre 40 et $50 \mathrm{~m} . \mathrm{s}^{-1}$. À l'intérieur des zones en grisé, la vitesse du vent est supérieure à $50 \mathrm{~m} \cdot \mathrm{s}^{-1}$ L'axe du courant-jet sur la surface $300 \mathrm{hPa}$ est représenté par un trait épais continu fléché. D1, D2, C1, C3 sont respectivement les maxima de divergence et de convergence (données du CEPMMT). en aval du thalweg d'altitude, était systématiquement inhibée par les effets de courbure du flux (Cammas et Ramond, 1989). Ainsi, le mécanisme à l'origine de la foliation de tropopause, les 2 et 3 juin 1984, est la circulation agéostrophique longitudinale induite par l'advection de courbure le long du flux. Ce dernier mécanisme des foliations de tropopause n'avait pas fait, à notre connaissance, l'objet d'une vérification par analyse diagnostique.

Ces résultats constituaient une première étape vers l'étude des couplages dynamiques par les circulations agéostrophiques entre les fronts d'altitude et les fronts de surface. Dans ce but, une étape indispensable, vu les échelles spatiotemporelles des phénomènes étudiés, était l'intégration dans les analyses de données de mésoéchelle. Ce travail a été abordé dans le cadre de LandesFronts 84 avec les données du radar ST Provence en configuration de tir vertical. L'analyse de ces données a montré qu'il n'est pas immédiat de filtrer dans les spectres de vitesses verticales des signatures de processus de méso-échelle, tels que les ondes de gravité, pour en tirer une signature d'un processus de méso-échelle, tel que celui d'une circulation agéostrophique. Ce travail est poursuivi dans le cadre des études sur l'expérience Fronts 87.

\section{ÉTUDE DES ONDES DE GRAVITÉ}

L'expérience Landes-Fronts 84 a été l'occasion de compléter l'étude des ondes de gravité par radar ST entreprise en 1982 lors de la campagne Alpex (Carter et al., 1989 ; Ecklund et al., 1985), puis de les poursuivre sur le même site pendant la campagne MésoGers (Ralph et al., 1993a). Ces campagnes représentaient la première occasion d'introduire un radar ST dans une campagne multi-instrumentale et dans un environnement météorologique beaucoup mieux documenté que lors de la campagne Alpex. C'était aussi la possibilité d'étudier parallèlement la physique de la mesure à l'aide d'observations in situ à haute résolution (Dalaudier et al., 1989). Malgré une configuration réduite du système radar, des mesures permanentes à haute résolution temporelle ont été effectuées sur les profils de vitesse verticale de l'air et sur l'évolution de l'altitude de la tropopause.

Lors des situations perturbées, de nombreux phénomènes ondulatoires ont été mis en évidence :

- Les ondes de relief, étudiées lors de la campagne Alpex (Ecklund et al., 1985) et plus récemment à Toulon par radar ST (Ralph et al., 1992), ont été observées par flux de sud mais n'ont pas fait l'objet d'études particulières pour cette campagne. Les résultats obtenus sont en cours d'intégration dans une étude plus générale.

- Certaines ondes de gravité (libres ou guidées) non observées sur le réseau sol ont pu être étudiées à l'aide du seul radar ST. C'est en particulier le cas d'un certain nombre



Figure 9 - Profils verticaux de la vitesse du vent parallèle à l'onde, déterminés à partir de radiosondages aux heures indiquées, le 19 juin 1984. La vitesse de l'onde $\left(14,1 \mathrm{~m} . \mathrm{s}^{-1}\right)$ est repérée ainsi que l'erreur (1,2 m.s. sur la vitesse de phase au sol. 
d'ondes guidées observées lors de la période du $1^{\text {er }}$ au 4 juin 1984 (Brun, 1990). L'analyse de ces ondes s'appuyant sur les mesures radar et les radiosondages a permis d'identifier l'origine du guide et de l'associer à la structure du profil


vertical de la vitesse horizontale du vent comme proposé par Chimonas et Hines (1986).

- Certains phénomènes ondulatoires ont été simultanément observés par radar et au sol (figure 8) par le réseau de barographes 4M. L'étude conjuguée radar ST-réseau sol permet alors une description complète des caractéristiques de l'onde (période, longueur d'onde, vitesse de phase et structure verticale). De telles observations complètes et coordonnées d'ondes de gravité à méso-échelle ont rarement été publiées dans la littérature, car les résultats présentés antérieurement étaient confinés à des mesures de surface sans information directe sur la structure verticale. L'onde observée le 19 juin 1984 était guidée dans une atmosphère où la stabilité statique répondait aux conditions introduites par Lindzen et Tung (1976) et présentait donc des caractéristiques favorables au guidage. Le niveau critique (où la vitesse de phase de l'onde est égale à la composante du vent parallèle à l'onde) était présent (figure 9) juste au-dessus d'une couche de faible stabilité statique surmontant une couche plus stable à un niveau inférieur (figure 10). L'onde (de $90 \mathrm{mn}$ de période, de $76 \mathrm{~km}$ de longueur d'onde horizontale et de $14 \mathrm{~m} / \mathrm{s}$ de vitesse orientée à $50^{\circ}$ vers le nord-est) a été observée lors d'une intense activité météorologique associée à une ligne de grains. Cependant, des conclusions définitives n'ont pu jusqu'alors être obtenues sur l'interaction entre l'onde et la convection locale enregistrée grâce au réseau sol (Ralph et al., 1993b).

Figure 10 - Profils verticaux d'indice de stabilité statique $\mathrm{N}^{2}$ obtenus à partir des radiosondages sur la zone de l'expérience et calculés en tenant compte de l'humidité, le 19 juin 1984. La couche stable qui forme un guide à basse altitude est indiquée, ainsi que les niveaux où la vitesse de phase de l'onde est égale à la vitesse parallèle du vent (flèches).

ÉTUDES MICROPHYSIQUES
Les avions Piper Aztec et Transall ont été équipés pour faire des mesures dynamiques, microphysiques et électriques. Des mesures de granulométrie des précipitations ont été effectuées au sol à partir du spectropluviomètre Vidiaz. Des résultats originaux ont ainsi été obtenus sur les processus de condensation, la multiplication des cristaux de glace, la représentation statistique des champs microphysiques et la granulométrie des précipitations en fonction de la phase de croissance des nuages convectifs.

L'expérience Landes-Fronts 84 fournissait la première opportunité de tester in situ le système de mesures microphysiques installé sur l'avion Piper Aztec. Le programme de recherche visait à documenter séparément quelques aspects particuliers du processus de formation des gouttelettes de nuage par condensation. $\mathrm{Au}$ terme de l'étude, les objectifs affichés ont été largement dépassés. Les mesures effectuées ont en particulier permis de décrire le processus de condensation dans sa globalité et d'en proposer une paramétrisation originale (Brenguier, 1990 et 1991).

La confirmation expérimentale des schémas existants nécessitait une approche nouvelle des mesures de granulométrie et, en particulier, l'analyse précise des effets de coïncidence dans le compteur de gouttelettes. Ce problème statistique, qui n'avait été traité jusqu'alors que de manière approximative, a pu être résolu de manière formelle (Brenguier et Amodei, 1989). La résolution complète des équations statistiques fournit ainsi une évaluation précise du taux de coïncidence à l'intérieur d'un échantillon et permet à la fois de vérifier l'homogénéité des échantillons de gouttes et d'évaluer (figure 11) leur concentration locale (Brenguier, 1989 ; Brenguier et al., 1994). La méthode est aussi le seul moyen objectif de détecter, à posteriori, des défauts de fonctionnement en vol de la sonde, tels que le givrage de l'optique (Brenguier et al., 1993). Cette théorie 
Figure 11 - Histogramme des valeurs de la concentration (par mg d'air) des gouttelettes (diamètre inférieur à $50 \mu \mathrm{m}$ ) dans un cumulus non précipitant échantillonné le 28 juin 1984. En (a), les valeurs mesurées, en (b) les valeurs calculées après correction des coïncidences et des hétérogénéités.

Cette figure montre que les valeurs fortes de la concentration (entre 800 et $1200 \mathrm{mg}^{-1}$ ) sont très sous-estimées à cause des pertes par coïncidence, les valeurs réelles se situant entre 1000 et $1500 \mathrm{mg}^{-1}$, et que le fort pourcentage de valeurs faibles mesurées (entre 0 et $300 \mathrm{mg}^{-1}$ ) disparaît après calcul des valeurs locales. Cela signifie que dans les régions hétérogènes (mélange de ciel clair et de nuages), la concentration locale (dans la partie nuageuse de l'échantillon) est la même que dans le reste du nuage. Cette observation implique que le mélange ne conduit pas à une dilution de la concentration des gouttes et que, localement, la concentration reste inchangée ; le mélange est hétérogène (d'après Brenguier, 1990).
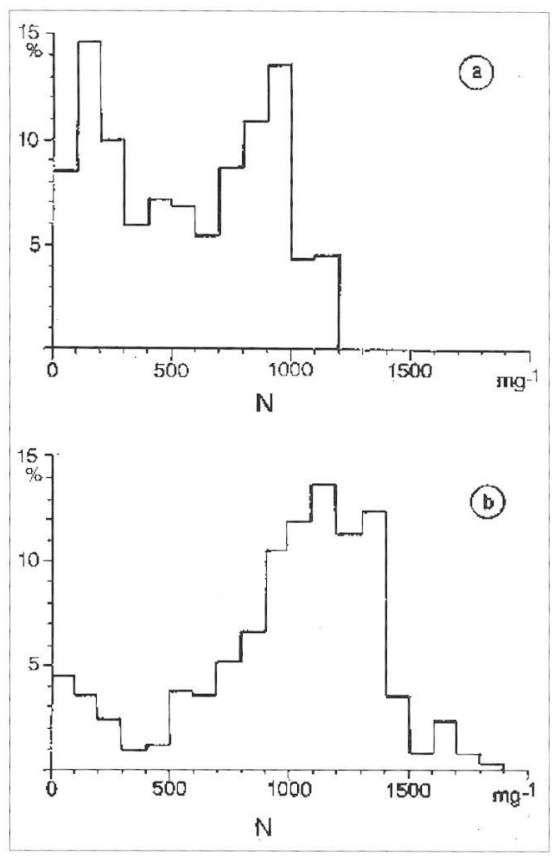

statistique a ouvert de nouvelles perspectives. D'une part, elle a permis la mise en évidence des hétérogénéités de petite échelle dans les zones de mélange des nuages convectifs observés pendant Landes-Fronts 84, observations qui sont essentielles pour la compréhension des processus de croissance des gouttes. D'autre part, une nouvelle électronique plus performante pour le granulomètre a été conçue (Brenguier et al., 1995) et a permis d'affiner considérablement les premières observations (Brenguier, 1993).

Partant de ces résultats expérimentaux, un schéma de paramétrisation a été développé pour les processus de condensation (Brenguier, 1990); il permet de reproduire l'évolution verticale des spectres de gouttes et leur distribution statistique dans un nuage convectif.

La méthode a ensuite été généralisée (Brenguier, 1991). On a montré, tout d'abord, que la distribution dimensionnelle d'une population de gouttes suivant la même trajectoire (population élémentaire) peut être décrite simplement à partir d'un seul paramètre, à savoir l'intégrale de la sursaturation le long de cette trajectoire ; les larges distributions observées peuvent être représentées comme des combinaisons linéaires de ces populations élémentaires. La résolution du problème de la croissance des gouttes dans un nuage convectif se limite alors à la détermination en tout point de la fonction de densité du paramètre. Cette approche est appropriée, car la détermination expérimentale de la fonction de densité du paramètre est possible à partir de n'importe quelle mesure de distribution dimensionnelle des gouttes et parce qu'elle permet d'obtenir des solutions simples du système d'équations non linéaires qui régit la croissance des gouttes. Ces solutions se substituent au schéma traditionnel de simulation numérique unidimensionnelle à microphysique détaillée. On a ainsi obtenu des solutions analytiques approchées pour l'étude préliminaire de nombreux processus : mélange d'entraînement, fluctuations de l'altitude de la base, fluctuations de la concentration des gouttes. La méthode est également bien adaptée à la simulation multidimensionnelle du nuage, parce qu'elle permet de simplifier le schéma microphysique dans le modèle et facilite l'interprétation des résultats obtenus (Brenguier et Grabowski, 1993).

Les mesures effectuées, le 6 juin 1984, à partir de l'avion Transall 04 dans des nuages convectifs (Laroche et al., 1985), ont permis de mettre en évidence, autour du niveau $-6{ }^{\circ} \mathrm{C}$, des concentrations de cristaux de glace $\left(600 \mathrm{l}^{-1}\right)$ très largement supérieures à celles issues de mécanismes primaires de formation de cristaux de glace (Gayet et Soulage, 1992). Les conditions thermodynamiques et microphysiques rencontrées étaient favorables à la formation secondaire de cristaux par splintering ${ }^{(1)}$. Cependant, les taux de production calculés se sont révélés inférieurs à ceux observés, suggérant ainsi l'intervention d'autres processus de multiplication des cristaux de glace (nucléation à sursaturations élevées) efficaces au cours du stade initial de glaciation des cellules convectives.

L'analyse des mesures in situ à petite échelle spatiale $(100 \mathrm{~m})$ a aussi montré que les processus de multiplication des cristaux de glace sont efficaces uniquement sur les bords des courants ascendants des cellules convectives (figure 12). L'apparition soudaine et massive de ces cristaux coïncide avec la phase dissipative du courant ascendant. À partir de ces observations, grâce à une évaluation des flux de chaleur latente libérée par givrage et par déposition de vapeur, on

(1) Sous certaines conditions thermodynamiques, les gouttelettes d'cau surfondue captées par un cristal de glace se congèlent en se fragmentant pour donner plusieurs cristaux de petites dimensions (approximativement $5 \mu \mathrm{m}$ ). Ce phénomène nommé «splintering» a été mis en évidence par des expériences en laboratoire (Hallet et Mossop, 1974). 




suggère que les cellules convectives s'auto-ensemencent massivement de cristaux de glace par mélange et recirculation, et que la glaciation très rapide qui en résulte n'apporte pas de modification sensible à la structure dynamique des cellules.

Par ailleurs, l'ensemble des mesures microphysiques réalisées à bord de l'avion Transall a été utilisé pour la caractérisation statistique des conditions givrantes, travail réalisé en collaboration avec la Federal Aviation Administration (FAA) des États-Unis (Gayet, 1986).

Figure 12 - Série temporelle des paramètres microphysiques et de la vitesse verticale du vent (WA) obtenue au cours de la traversée d'un nuage convectif au niveau $-6{ }^{\circ} \mathrm{C}$, le 6 juin 1984.

$\mathrm{CH}$ : concentration des particules de glace de dimension supérieure à $250 \mu \mathrm{m}$. $\mathrm{Cl}$ : concentration des particules de glace de dimension inférieure à $50 \mu \mathrm{m}$. LWC : contenu en eau liquide.

La région repérée en $\mathrm{A}$ correspond à une zone nuageuse dépourvue de cristaux de glace ; au contraire, les régions B dénotent les zones ayant une forte concentration en cristaux (d'après Gayet et Soulage, 1992).

\section{Paramétrisation de la microphysique}

La difficulté d'utiliser seules les données microphysiques, pour l'étude de la physique des précipitations, nous a conduit à réfléchir au problème de l'hétérogénéité des champs microphysiques et à leur représentation statistique ; c'est l'un des thèmes importants dans la paramétrisation des caractéristiques de nuages à une échelle inférieure à la maille des modèles numériques. L'approche adoptée pour ce problème consiste à examiner l'applicabilité d'outils analytiques développés récemment dans la géométrie fractale.

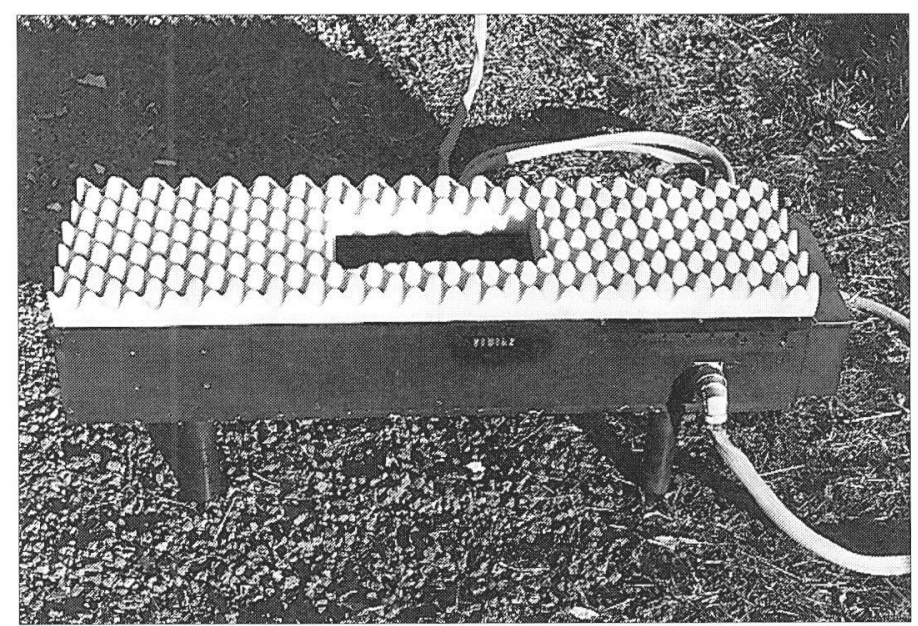

C'est dans ce but que les données des expériences Landes-Fronts 84 ont été utilisées, en plus de celles de Nephos 82 et, plus récemment, de Fronts 87. L'analyse expérimentale montre l'existence d'une propriété d'invariance d'échelle dans les zones nuageuses et non nuageuses pour les échelles inférieures à l'échelle verticale de nuages. La question qui se pose maintenant est de savoir s'il existe une propriété d'invariance d'échelle aux échelles supérieures, comme le montrent différents travaux s'appuyant sur l'analyse d'images satellitales. La seconde question (Duroure et Guillemet, 1990) est de savoir comment générer un champ de nuages à partir de statistiques unidimensionnelles et quelles sont les hypothèses nécessaires à cette génération.

Partie «capteur» du spectropluviomètre Vidiaz mis en œuvre par l'Observatoire de physique du globe de Clermont-Ferrand. Cet instrument fournit la distribution en taille des gouttes de pluie.

\section{Analyse granulométrique des précipitations}

Une averse isolée issue d'une cellule convective a été enregistrée au sol à l'aide du spectropluviomètre Vidiaz (Donnadieu, 1978) le 1 1er juin 1984. L'analyse granulométrique en a été faite par Donnadieu (1988) en utilisant la paramétrisation de Waldvogel (1974), qui considère une distribution exponentielle des gouttes $\mathrm{n}(\mathrm{D})=\mathrm{N}_{0} \mathrm{e}^{-\lambda \mathrm{D}}$ ayant la même réflectivité et la même teneur en eau que la distribution observée.

La figure 13a montre, en fonction du temps, l'intensité de précipitation $\mathrm{R}$ et les deux paramètres de distribution $\mathrm{N}_{0}$ et $\lambda$. On met en évidence une phase de croissance (points 1 à 8) suivie d'une phase de dissipation (points 9 à 16). La première est caractérisée par de faibles valeurs de $\mathrm{N}_{0}$ et de $\lambda$, c'est-à-dire par des spectres de distribution des gouttes de type exponentiel plutôt larges, tandis que la seconde l'est par de fortes valeurs de $N_{0}$ et de $\lambda$, c'est-à-dire par des spectres plus étroits. Le passage de la phase de croissance de l'averse à sa phase de dissipation se traduit, sur une durée assez brève, par une augmentation importante des deux paramètres $\mathrm{N}_{0}$ et $\lambda$. Cette période de changement de phase (ou phase transitoire) peut être caractérisée par un spectre moyen de type exponentiel à double pente (Donnadieu, 1985). 

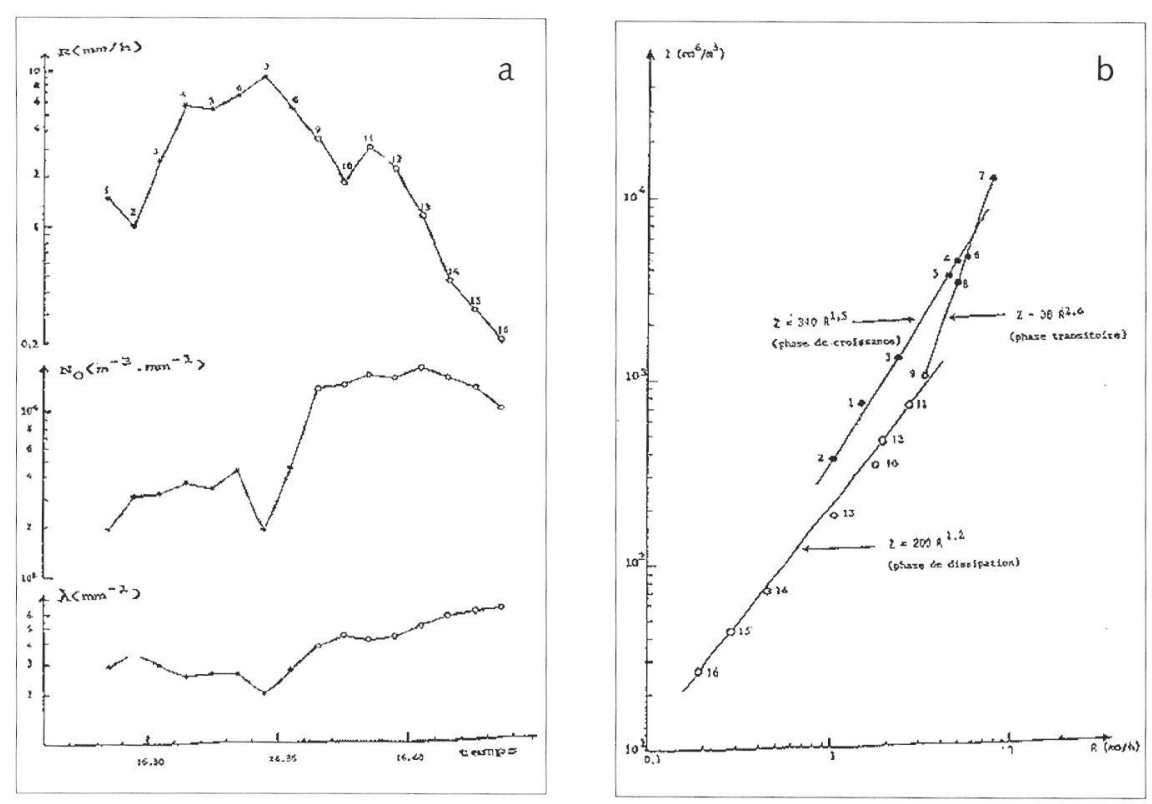

Figure 13 -

a) Intensité de précipitation $R$ et paramètres de distribution des gouttes $N_{0}$ et $\lambda$ en fonction du temps pour l'averse du $1^{\text {er }}$ juin 1984 $(\bullet=1$ à 8 , phase de croissance ; $0=9$ à 16 , phase de dissipation).

b) Représentation des différentes relations $Z=A R^{b}$ observées pendant les phases de I'averse.

C) Relations Z-R obtenues par Carbone et Nelson (1978) lors de mesures à la base de nuages convectifs en phase de croissance et de dissipation. Les croix représentent, en fonction du temps, les résultats de leur modèle numérique.

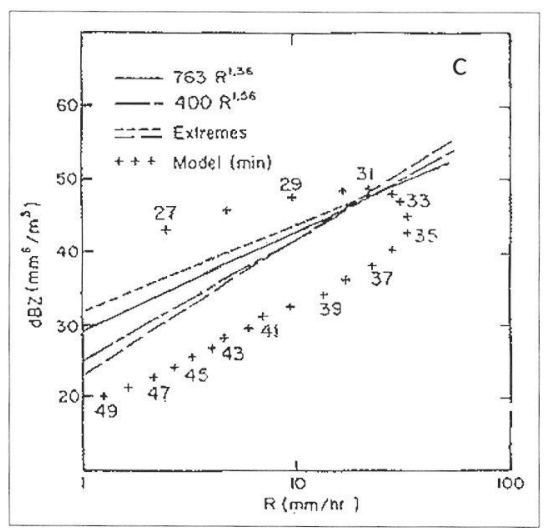

Du point de vue des relations classiques $\mathrm{Z}=\mathrm{AR}^{\mathrm{b}}$, où $\mathrm{Z}$ est la réflectivité, la phase de croissance est caractérisée par un coefficient A presque double de celui de la phase de dissipation ; pour la phase transitoire, les deux coefficients A et b présentent des valeurs très inhabituelles (figure 13b). On observe enfin que, dans cette représentation $\mathrm{Z}-\mathrm{R}$, les points de mesure décrivent, en fonction du temps, une forme de boucle : ces résultats obtenus au sol sont comparables à ceux mis en évidence par Carbone et Nelson (1978) lors de mesures granulométriques à la base des nuages convectifs et vérifient leur modèle numérique (figure 13c).

ÉTUDE DES PHÉNOMÈNES ÉLECTRIQUES

\section{Représentativité des mesures de champ électrique de surface}

L'étude des phénomènes électriques a largement bénéficié, dans cette expérience, de la complémentarité des moyens de mesure disponibles, tant au sol qu'en altitude. Des résultats originaux ont pu être obtenus, en particulier, sur le foudroiement des avions, sur les interactions entre phénomènes microphysiques et phénomènes électriques, ainsi que sur la localisation respective des décharges et des précipitations.

Des mesures de champs électriques étaient effectuées au sol par un moulin à champ étalon et à 15 mètres au-dessus, en point fixe, grâce à la mise en œuvre d'une sonde isolée de la terre et fonctionnant par télémesure. Elles ont permis de caractériser le comportement et le rôle de la couche de charge d'espace qui se forme au-dessus du sol sous orage. Cette couche est constituée d'ions produits par effet Corona sur les irrégularités de surface lorsque le champ local dépasse un certain seuil d'intensité.

L'étude met tout d'abord en évidence l'influence considérable, sur le champ électrique de surface, de cette couche de charge. Ainsi, la figure 14, représentant un résultat de la mesure simultanée du champ électrique aux deux hauteurs, 




Figure 14 - Variations temporelles, lors de l'orage du 7 juillet 1984 , du champ électrique au sol (1) et à $15 \mathrm{~m}$ de hauteur (2), ainsi que de la densité volumique de charge (3) calculée par l'équation de Poisson. montre à quel point l'intensité de surface est profondément affectée (essentiellement réduite) par la présence et l'évolution de cette charge d'espace. En outre, l'application de l'équation de Poisson ( $\left.\operatorname{div} E=\rho / \varepsilon_{0}\right)$ à ces résultats, permet de déterminer l'évolution d'une densité volumique moyenne dans la couche $0-15 \mathrm{~m}$ qui atteint $6 \mathrm{nC} / \mathrm{m}^{3}$.

On a également pu montrer qu'entre les éclairs, le champ électrique mesuré au sol se stabilise à une valeur limite de l'ordre de quelques $\mathrm{kV} / \mathrm{m}$. Lorsqu'il en est ainsi, sa variation due aux charges nuageuses est exactement compensée par celle provoquée par la production locale d'ions corona. Cette valeur limite dépend du taux de production ionique ainsi que du taux de régénération des charges nuageuses (Chauzy et Soula, 1987).

Ainsi, près du sol, une variation considérable du champ électrique en fonction de l'altitude est provoquée par la présence de la couche de charge d'espace. Les écarts observés atteignent $35 \mathrm{kV} / \mathrm{m}$ entre le sol et une altitude de $500 \mathrm{~m}$, et près de $10 \mathrm{kV} / \mathrm{m}$ entre le sol et $15 \mathrm{~m}$. Les résultats indiquent également que les plus grandes densités de charge se situent près de la surface. Les sondages de champ, les mesures effectuées au voisinage du sol ainsi que la modélisation confirment ce phénomène (Chauzy et Soula, 1987).

Le rôle des éclairs dans la production d'ions corona au sol est également mis en évidence. Ainsi, les mesures simultanées de champ électrique à deux hauteurs indiquent que le rétablissement rapide, au niveau du sol, du champ électrique après l'éclair est essentiellement dû à la production locale d'ions corona, contrairement à ce qu'affirment certaines théories qui attribuent cette variation à des mécanismes intranuage. Enfin, les ions corona ont une influence sur la variation du champ électrique, même pendant la courte durée d'un éclair (Soula and Chauzy, 1986 ; Chauzy et al., 1989).

Tous ces résultats ont pu être vérifiés sur le plan théorique en mettant en œuvre un modèle numérique d'évolution des ions corona (modèle Picasso) afin de confirmer les hypothèses émises après examen des résultats expérimentaux, d'étudier les influences relatives des divers mécanismes et de suggérer des solutions aux problèmes posés.

Structures électrostatiques des cellules orageuses

La mise en œuvre de sondages de champ électrique effectués par ballon libre et sondes fonctionnant par télémesure constitue le moyen d'accéder à la structure électrostatique des cellules orageuses. En effet, on peut admettre, en première approximation, que le barycentre d'une zone chargée se situe à l'altitude à laquelle s'annule la composante verticale du champ électrique ambiant. La détection de cette composante, au cours de l'ascension du ballon, donne donc accès à la répartition verticale de ces zones.

La faible activité électrique localisée au-dessus du site de lancement des ballons (Luppé-Violles) n'a permis d'effectuer que deux sondages exploitables : le 12 mai et le 21 juin 1984. Le premier a permis d'enregistrer des valeurs maximales du champ de l'ordre de $100 \mathrm{kV} / \mathrm{m}$ dans les basses couches, alors que le second (figure 15) traduit une distribution globalement dipolaire de l'accumulation de charge. La zone chargée négativement se situe autour de $3700 \mathrm{~m}$ - isotherme $-4{ }^{\circ} \mathrm{C}$ - et la zone positive autour de $5400 \mathrm{~m}$ - isotherme $-14{ }^{\circ} \mathrm{C}$ (Soula et Chauzy, 1986).

L'analyse des observations in situ effectuées à partir du Transall (Gondot, 1988) a permis de mettre en évidence un centre positif de basse altitude et d'étudier le mécanisme responsable de cette formation, à savoir les collisions «particules de neige roulée-cristaux de glace» en présence de gouttelettes d'eau surfondue. Cette analyse a été complétée (Laroche et al., 1986) pour montrer l'association de ce phénomène avec la production secondaire de cristaux de glace par splintering. En plus de ces analyses détaillées sur la nature des particules chargées et le mécanisme de génération de charge, on a pu déterminer les caractéristiques microphysiques, électriques et dynamiques de zones favorables au foudroiement d'un avion dans un orage (Gayet et al., 1985).

Parallèlement à ces travaux expérimentaux, l'intégration des mécanismes de génération des charges électriques dans un modèle diagnostique de nuage a été entreprise (Chen Fei, 1990). Ce travail montre que les processus microphysiques 


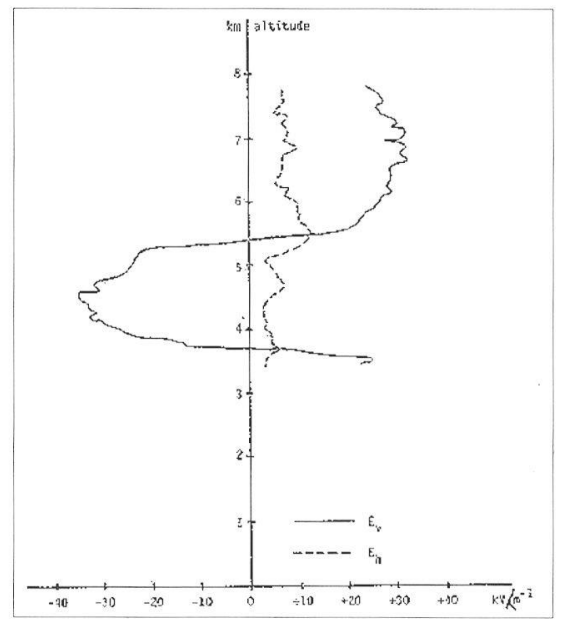

intervenant dans la génération et la redistribution des charges électriques sont suffisamment efficaces pour donner rapidement une distribution de charges électriques en équilibre quasi stationnaire. Ce résultat permet alors de normaliser cette distribution en fonction des champs électriques et des taux de précipitation.

Figure 15 - Sondage de champ électrique du 21 juin 1984, à O h 20 UTC.

$E_{v}$ représente la composante verticale, $E_{h}$ la composante horizontale du champ.

\section{Propriété des décharges et comparaison avec les données radar}

\section{Étude du foudroiement d'un avion}

Le but de ces études était de caractériser l'activité électrique de manière à identifier et analyser les processus élémentaires intervenant dans le développement des décharges. On cherchait également à établir des comparaisons entre la localisation des décharges et celles des précipitations en fonction de la dynamique des cellules.

L'analyse a été conduite principalement à l'aide d'un interféromètre VHF tridimensionnel, fonctionnant à $300 \mathrm{MHz}$, avec une résolution temporelle d'une microseconde.

Les propriétés des différentes composantes (phases, «leader» et arc en retour) des éclairs ont été analysées (Richard et al., 1986). Des systèmes qui se déplacent à $2.10^{7} \mathrm{~m} / \mathrm{s}$ sur de grandes distances (plusieurs kilomètres) ont été identifiés pour la première fois. Ils correspondent à des décharges de jonction se propageant dans les canaux des leaders positifs qui se développent initialement depuis la zone d'amorçage de l'éclair. Ces mesures ont permis de visualiser la propagation bidirectionnelle des décharges dans le nuage. Ce concept n'avait jamais pu être validé auparavant.

Par comparaison avec les données radar, on a pu montrer que la cadence des éclairs, leur longueur et le rapport entre éclairs intranuage et nuage-sol dépendent de la phase de développement d'une cellule. L'activité nuage-sol apparaît après le début de l'effondrement dynamique. Les zones d'activité principale correspondent à celles de forte réflectivité radar.

Cette étude avait pour but de caractériser un foudroiement en vol, d'analyser son couplage avec les circuits de l'avion et d'étudier l'environnement atmosphérique au moment de l'impact. L'expérience était réalisée à l'aide d'un avion instrumenté (Transall A04 du Centre d'essais en vol).

Nous avons pu montrer que dans la majorité des cas $(90 \%)$, l'avion déclenche l'éclair en émettant un leader positif puis une décharge bidirectionnelle. Les courants associés aux phases les plus intenses des éclairs intranuage sont typiquement inférieurs à $20 \mathrm{kA}$. L'étude a permis de modéliser ce phénomène qui a été appliqué à la mise au point de codes numériques.

Les foudroiements ont été obtenus pour des valeurs modérées du champ atmosphérique (40 à $60 \mathrm{kV} / \mathrm{m}$ ). La teneur en eau liquide et la présence de cristaux ne semblent pas influencer notablement la probąbilité de déclenchement de l'éclair par l'avion. En revanche, on a pu mettre en évidence une augmentation systématique de la conductivité pendant les secondes précédant l'éclair. Les ions ne semblent pas être originaires de l'avion. Dans ce cas, ils traduisent un conditionnement du milieu nuageux favorisant le déclenchement de la foudre. Enfin, on a remarqué que le foudroiement de l'avion se produisait fréquemment à la sortie de zones de précipitations chargées. Il est probable que les mêmes conditions électrostatiques existent à l'entrée de ces zones. Le fait que l'on n'observe jamais de foudroiement en entrée de ces zones résulte peut-être de ce que l'éclair vide ces poches de charge, la zone chargée disparaissant alors juste avant que l'avion ne la pénètre. L'ensemble de ces observations enrichit notablement notre connaissance de la phénoménologie du foudroiement en vol et, plus généralement, du déclenchement de l'éclair. 
ACQUIS MÉTHODOLOGIQUES ET LOGISTIQUES

\author{
Restitution du champ \\ de vent tridimensionnel \\ à moyenne échelle
}

Les acquis méthodologiques et logistiques obtenus à la suite d'une expérience comme Landes-Fronts 84 sont nombreux ; nous présentons ici ceux qui nous sont apparus rapidement exploitables à l'issue de l'expérience.

La restitution du champ de vent tridimensionnel à moyenne échelle est apparue comme une nécessité pour l'étude des circulations de moyenne échelle dans les systèmes frontaux. Une nouvelle méthode d'analyse des champs de vitesses radiales issus de (au moins) deux radars Doppler a ainsi été développée, à l'aide des données de Landes-Fronts 84 (Scialom et Lemaître, 1990). Dans cette méthode appelée Mandop (Multiple Analytical Doppler), chaque composante du vent est supposée s'exprimer sous la forme d'un produit de trois développements en série de fonctions orthonormales, chaque fonction dépendant d'une seule coordonnée d'espace. On peut ainsi également exprimer la vitesse radiale vue par chaque radar sous cette forme. Afin de déterminer les coefficients inconnus, cette forme analytique du vent radial est ajustée au sens des moindres carrés aux vents radiaux observés et, de plus, contrainte à vérifier sous forme variationnelle l'équation de continuité et la condition au sol (vitesse verticale nulle). La méthode Mandop permet également la restitution des champs de perturbation de pression et de température au moyen de l'utilisation de l'équation du mouvement. La restitution du champ de vent tridimensionnel par cette méthode a pu être menée à bien pour un cas d'observation de Landes-Fronts 84 . Il concerne un système convectif postfrontal (4 juin 1984) pour lequel l'organisation en bandes des précipitations résulte de la circulation de l'air sous forme de rouleaux de moyenne échelle, due à l'Instabilité symétrique conditionnelle (ISC).

Les difficultés rencontrées au cours de l'interprétation des données de LandesFronts 84 sont liées à l'insuffisance des informations disponibles à moyenne échelle, et ont conduit à mettre sur pied un dispositif plus complet pour Fronts 87 , avec un réseau de moyenne échelle comportant trois stations de radiosondage, trois radars ST, trois sodars et les deux radars Doppler Ronsard. Ce dispositif a pu être complété par un renforcement du réseau synoptique des radiosondages

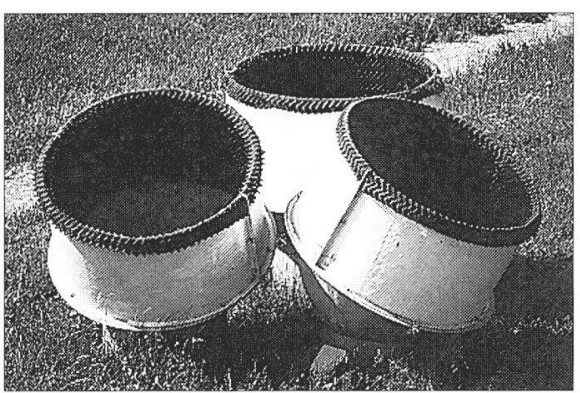
anglais et français et par le lâcher de dropsondes espacées de quelques dizaines de kilomètres.

\section{Mesure des hétérogénéités de petite échelle dans les nuages}

Les méthodes statistiques développées à 1'occasion de Landes-Fronts 84 pour évaluer les coïncidences se sont aussi révélées des outils puissants pour analyser les défauts des sondes granulométriques. En particulier, grâce à la réalisation d'un générateur d'impulsions aléatoires poissonnien, on a pu montrer que les défauts attribués à de mauvais réglages de l'électronique ou de l'optique de la sonde utilisée étaient en fait inhérents au principe de mesure (Brenguier, 1989). C'est ainsi qu'une nouvelle chaîne électronique a été conçue pour pallier ces inconvénients et étendre le domaine de fonctionnement des sondes granulométriques FSSP(1) aux vitesses de vol des nouveaux avions de recherche Merlin et Arat (Brenguier et al., 1995). Enfin, une procédure a été développée pour détecter des dégradations de la mesure, comme celles qui peuvent résulter du givrage de l'optique de la sonde (Brenguier et al., 1993). Sans le recours aux équations des coïncidences, les sections de vol où la mesure est dégradée seraient acceptées, ce qui conduirait à des interprétations erronées. Cette procédure, utilisable en temps réel à bord de l'avion, permet d'interrompre une expérience pour redescendre, dans le cas de givrage, à température positive jusqu'à ce que la sonde ait recouvré ses performances nominales. 
L'étude a ensuite pu être poursuivie grâce à des financements du Centre national de recherches météorologiques, du Comité des Avions de recherche atmosphérique et de télédétection (ARAT) et du Programme atmosphère moyenne et océan superficiel (PAMOS). Le «Fast FSSP» a effectué son premier vol en juillet 1991 et a produit des données très originales à micro-échelle. Ces données ont permis, en particulier, de documenter des interfaces très étroites, d'une dimension inférieures au centimètre, entre le nuage et son environnement (Brenguier, 1993). Le prototype a été suivi d'une version opérationnelle embarquable sur n'importe quel avion équipé pour la microphysique et a participé à de nombreuses expériences internationales. Outre la mesure à micro-échelle, le Fast FSSP permet d'améliorer très nettement la précision des mesures de concentration et de diamètre des gouttes (Brenguier et al., 1995).

\section{CONCLUSION}

Cette expérience a permis d'acquérir une meilleure connaissance des caractéristiques dynamiques, microphysiques et électriques des précipitations qui ont affecté le Sud-Ouest de la France. Elle a été le point de départ d'une longue série de résultats originaux, aussi bien sur le plan instrumental que sur les plans expérimental et théorique. Elle a en particulier permis de comprendre l'influence de l'instabilité symétrique et des circulations en pente ou en rouleaux sur la formation de nuages convectifs et orageux dans des situations où le relâchement de l'instabilité convective paraissait improbable en raison de l'importance des freins à la convection rencontrés dans les basses couches (forte énergie d'inhibition convective et niveau de convection libre élevé).

De nombreux résultats ont aussi été obtenus sur la structure et les variations du champ électrique, leur formation et leur interaction avec les précipitations. Les conditions favorables au foudroiement d'un avion en vol et leur localisation dans les nuages ont ainsi pu être déterminées.

Les cas présentés à titre d'illustration ont confirmé l'importance des processus de moyenne échelle dans les systèmes convectifs observés à nos latitudes ; on a pu décrire certains des mécanismes d'interaction entre les échelles de mouvement et mettre en évidence le rôle important que jouent les processus microphysiques en phase glace dans l'intensification des circulations de moyenne échelle. Toutefois, l'interaction avec les phénomènes de grande échelle n'a pu être abordée que de façon qualitative, du fait de la présence des Pyrénées et de l'insuffisance des moyens disponibles, lors de l'expérience, pour appréhender ces échelles.

Il est évident que si les études qui ont été effectuées permettent de comprendre certaines caractéristiques particulières à des systèmes convectifs ayant affecté la région des Landes et du Gers, les restrictions imposées par la dimension de la zone d'observation du champ de vent (deux carrés de 50 × $50 \mathrm{~km}^{2}$ ) par les radars Doppler, d'une part, et par la durée de l'expérience (un peu plus de deux mois), d'autre part, ne permettent pas de généraliser ces observations à l'ensemble des systèmes susceptibles de se développer dans le Sud-Ouest de la France.

\section{Remerciements}

Nous tenons ici à remercier l'ensemble des personnes ayant participé à l'acquisition, au dépouillement et à l'interprétation des données. Une aide précieuse a été apportée pour la réalisation des radiosondages supplémentaires par l'École nationale de la météorologie (ENM, Toulouse), la station météorologique de Bordeaux et la station météorologique de Toulouse-Blagnac. La Direction interrégionale Sud-Ouest de Météo-France (DIR Sud-Ouest) assurait la mise en œuvre des stations Patac. Le support financier et logistique a été fourni par les organismes participants, l'Institut national des sciences de l'univers (INSU) et la Direction des recherches et études techniques (DRET).

\section{BIBLIOGRAPHIE}

Brenguier J.-L., 1989 : Coincidence and dead-time corrections for the particle counters. Part II : High concentration measurements with an FSSP. J. Atmos. Ocean. Technol., 6, 585-598.

Brenguier J.-L. et L. Amodei, 1989 : Coincidence and dead-time corrections for the particle counters. Part I : A general mathematical formalism. J. Atmos. Ocean. Technol., 6, 575-584.

Brenguier J.-L., 1990 : Parameterization of the condensation process in small non precipitating cumuli. J. Atmos. Sci., 47, 1127-1148.

Brenguier J.-L., 1991 : Parameterization of the condensation process : A theoretical approach. J. Atmos. Sci., 48, 264-282. 
Brenguier J.-L., 1993 : Observations of cloud microstructure at the centimeter scale. $J$. Appl. Meteor., 32, 783-793.

Brenguier J.-L. et W. W. Grabowski, 1993 : Cumulus entrainment and cloud droplet spectra : a numerical model within a two-dimensional dynamical framework. J. Atmos. Sci., $50,120-136$

Brenguier J.-L., A. R. Rodi, G. Gordon et P. Wechsler, 1993 : Real time detection of performance degradation of the FSSP probe. J. Atmos. Ocean. Technol., 10, 27-33.

Brenguier J.-L., D. Baumgardner et B. Baker, 1994 : A review and discussion of processing algorithms for FSSP concentration measurements. J. Atmos. Ocean. Technol., 11, 1409-1414.

Brenguier J.-L., D. Trévarin, R. Peytavi, A. Coelho, P. Wechsler and J. Isbert, 1995 : New electronics for the Forward Scattering Spectrometer Probe : the Fast FSSP. Accepté à $J$. Atmos. Ocean. Technol.

Brun E., 1990 : Ondes de gravité dans la basse atmosphère : étude expérimentale par radar ST. Thèse de doctorat, soutenue à l'université de Paris VI, le 9 mars 1990, 157 p.

Cammas J.-P., 1988 : Circulations agéostrophiques dans les systèmes jet-fronts : une étude prospective du couplage dynamique haute et basse troposphère. Thèse de doctorat, soutenue à l'université Blaise Pascal de Clermont-Ferrand, le 29 septembre 1988, 237 p.

Cammas J.-P. et D. Ramond, 1989 : Diagnosis and analysis of ageostrophic circulation in jet-front systems. Mon. Wea. Rev., 117, 11, 2447-2462.

Carbone R. E. et L. D. Nelson, 1978 : The evolution of raindrop spectra in warm-based convective storms as observed and numerically modeled. J. Atmos. Sci., 35, 2302-2314.

Carter D. A., B. B. Balsley, W. L. Ecklund, K. S. Gage, A. C. Riddle, R. Garello et M. Crochet, 1989 : Investigations of internal gravity waves using vertically directed wind profilers. J. Geophys. Res., 94, D6, 8633-8642.

Chalon J.-P., 1987 : Landes-Fronts 84 : une expérience d'étude de bandes de convection associées aux systèmes frontaux. La Météorologie $7^{\mathrm{C}}$ série, 16, 7-23.

Chalon J.-P. et M. Gilet, 1980 : Structure à moyenne échelle d'un front froid. Communications à la $\mathrm{VII}^{\circ}$ Conférence internationale sur la physique des nuages, Clermont-Ferrand, France, 599-602.

Chauzy S. et S. Soula, 1987: General interpretation of surface electric field variations between lightning flashes. J. Geophys. Res., 92, D5, 5676-5684.

Chauzy S., S. Soula et S. Despiau, 1989 : Ground coronae and lightning. J. Geophys. Res., 94, D11, 13115-13119.

Chen Fei, 1990 : Étude numérique des processus d'électrisation d'un nuage chaud. Thèse de doctorat de l'université Blaise Pascal, Clermont-Ferrand, 192 p.

Chimonas G. et C. O. Hines, 1986 : Doppler ducting of atmospheric gravity waves. $J$. Geophys. Res., 91, 1219-1230.

Chong M. et G. Jaubert, 1990 : A mid-latitude squall line in France. Atmos. Res., 25 , 129-147.

Dalaudier F., M. Crochet et C. Sidi, 1989 : Direct comparison between in situ and radar measurements of temperature fluctuation spectra : A puzzling result. Radio Sci., 24, 3, 311-324.

Donnadieu G., 1978 : Mesure de la vitesse terminale des gouttes de pluie au sol à l'aide du spectropluviomètre Vidiaz. J. Rech. Atmos., 12, 245-259.

Donnadieu G., 1985 : Spectropluviométrie : existence de distributions exponentielles à double pente pouvant provenir de couches nuageuses stratoconvectives. C. R. Acad. Sc. Paris, 301, 1239-1244.

Donnadieu G., 1988 : Landes-Fronts 84 : analyse granulométrique au sol d'une averse de pluie convective. Atmos. Res., 22, 251-263.

Ducrocq V. et J.-M. Willemet, 1988 : Impact de la résolution dans le modèle Péridot : deux études de cas à méso-échelle. Note de travail de l'ENM n²40, Météo-France, Toulouse, 231p.

Ducrocq V., 1993 : Adiabatic and viscous simulations of symmetric instability : structure, evolution and energetics. J. Atmos. Sci., 50, 23-42.

Duroure C. et B. Guillemet, 1990 : Analyse des hétérogénéités spatiales des stratocumulus et cumulus. Atmos. Res., 25, 331-350.

Ecklund W. L., B. B. Balsey, D. A. Carter, A. C. Ruddle, M. Crochet et R. Garello, 1985 : Observations of vertical motions in the troposphere using three closely-spaced ST radars. Radio Sci., 20, 6, 1196-1206. 
Gayet J.-F., C. Duroure, R. G. Soulage et P. Laroche, 1985 : Location of lightning strokes on aircraft in storm field with measured electrical, microphysical and dynamical properties. Proc. 10th International conference on lightning and static electricity, Paris, 10-13 juin 1985, 67-70.

Gayet J.-F., 1986 : LAMP's contribution to the new characterization of the aircraft icing environment. Rapport scientifique du LAMP ${ }^{\circ} 56$, Université Blaise Pascal, ClermontFerrand.

Gayet J.-F. et R. G. Soulage, 1992 : Observation of high ice particle concentrations in convective cells and cloud glaciation evolution. Quart. J. Roy. Meteor. Soc., 118, 177-190.

Gondot P., 1988 : L'électrisation des nuages orageux : étude d'un cas de centre positif de basse altitude par des moyens aériens in situ. Thèse de doctorat d'État soutenue le 20 janvier 1988 à l'université de Clermont-Ferrand, 125 p.

Hallet J. et S. C. Mossop, 1974 : Production of secondary ice particles during riming process. Nature, 249, 26-28.

Jaubert G., M. Chong and J.-P. Giudicelli, 1988 : A middle latitude squall line in France. Proc. 10th Int. Cloud Phys. Conf., Bad-Homburg, Allemagne, 666-668.

Laroche P., M. Dill, J.-F. Gayet et M. Friedlander, 1985 : In flight thunderstorm environmental measurements during the Landes 84 campaign. Proc. 10th International Conference on lightning and static electricity, Paris, 10-13 juin 1985, 59-66.

Laroche P., A. Delannoy, P. Gondot, F. Helloco and J.-F. Gayet, 1986 : Airborne observations of small scale $(<1 \mathrm{~km})$ fluctuations of microphysical, dynamical and electrical properties in convective clouds. Proc. 23rd Conference on radar meteorology and cloud physics, Snowmass, Co, USA, 22-26 septembre 1986, J285-J289.

Lefèvre J.-M., 1987 : Déclenchement de la convection en présence d'instabilité symétrique conditionnelle. Rapport de stage de DEA, Université P. Sabatier, Toulouse, 80 p.

Lemaître Y., 1987 : Phénomènes de moyenne échelle dans les systèmes frontaux. $L a$ Météorologie $7^{\mathrm{c}}$ série, 18, 25-37.

Lemaître Y., G. Scialom et P. Amayenc, 1989 : A cold frontal rainband observed during the Landes-Fronts 84 experiment : Mesoscale and small scale structure inferred from dual Doppler radar analysis. J. Atmos. Sci., 46, 2215-2235.

Lemaître Y. et P. Brovelli, 1990 : Role of a low level jet in triggering and organizing moist convection in a baroclinic atmosphere. A case study : 18 May 1984. J. Atmos. Sci., 47, $82-100$

Lemaître Y. et G. Scialom, 1992 : Three dimensional mesoscale circulation within a convective post-frontal system. Possible role of conditional symmetric instability for triggering convective motions. Quart. J. Roy. Meteor. Soc., 118, 71-99.

Lindzen R. S. et K. K. Tung, 1976 : Banded convective activity and ducted gravity waves. Mon. Wea. Rev., 104, 1602-1617.

Ralph F. M., M. Crochet et S. V. Venkateswaran, 1992 : A study of mountain lee waves using clear-air radar. Quart. J. Roy. Society, 118, 597-627.

Ralph F. M., C. Mazaudier, M. Crochet et S. V. Venkateswaran, 1993a : Doppler sodar and radar wind profiler observations of gravity wave activity associated with a gravity wave current. Mon. Wea. Rev., 121, 444-462.

Ralph F. M., M. Crochet et S. V. Venkateswaran, 1993b : Observations of a mesoscale ducted gravity wave. J. Atmos. Sci., 50, 3277-3291.

Richard P., A. Delannoy, G. Labaune et P. Laroche, 1986 : Results of spatial and temporal characterization of UHF-VHF radiation of lightning. J. Geophys. Res., 91, 1.

Scialom G. et Y. Lemaitre, 1990 : A new analysis for the retrieval of three dimensional mesocale wind fields from multiple Doppler radar. J. Atmos. Ocean. Technol., 7, 640-665.

Soula S. et S. Chauzy, 1986 : The effects of ground coronae during lightning flashes. Ann. Geophysicae, 4B, 6, 613-624.

Testud J., G. Breger, P. Amayenc, M. Chong, B. Nutten et A. Sauvaget, 1980 : A Doppler radar observation of a cold front : Three-dimensionnal air circulation, related precipitation and associated wavelike motions. J. Atmos. Sci., 37, 78-98.

Trraff M., 1988 : Étude de la convection nuageuse dans le Sud-Ouest de la France, cas du 2 juin 1984 : effet du relief pyrénéen. Thèse de doctorat, soutenue le $1^{\text {er }}$ février 1988 à l'université de Clermont-Ferrand, 149 p.

Waldvogel A., 1974 : The No jump of raindrop spectra. J. Atmos. Sci., 31, 1067-1078. 
AUTRES Brenguier J.-L., 1991 : Étude expérimentale et paramétrisation des processus de condenPUBLICATIONS sation dans les cumulus non précipitants. Thèse de doctorat d'État, soutenue le 14 juin 1991 à l'université Blaise Pascal de Clermont-Ferrand.

Gayet J.-F., 1988 : Amélioration et emploi des techniques optoélectroniques pour la connaissance des paramètres microphysiques des nuages et du givrage atmosphérique Thèse de doctorat d'État, soutenue le 24 juin 1988 à l'université de Clermont-Ferrand, 209 p.

Gondot P., 1985 : Définition et exploitation de capteurs adaptés à la mesure de la conductivité électrique dans les nuages d'orages. Thèse de $3^{e}$ cycle, soutenue le 18 janvier 1985 à l'université de Paris VI, 87 p.

Laroche P., 1986 : Airborne measurements of electrical atmospheric field produced by convective clouds. Rev. Phys. Appl., 21, 809-815.

Ralph F. M. , 1991 : Mesoscale studies using clear-air Doppler radar. PhD Thesis, Department of Atmospheric Sciences, UCLA, Los Angeles, Ca, USA.

Scialom G. et J. Testud, 1986 : Retrieval of horizontal wind field and mesoscale vertical vorticity in stratiform precipitation by conical scannings with two Doppler radars. J. Atmos. Ocean. Technol., 3, 4, 693-703.

Soula S., 1986 : Influence de l'effet couronne sur le champ électrique mesurée au voisinage du sol sous orage. Thèse de doctorat de l'université Paul Sabatier, Toulouse, $214 \mathrm{p}$.

Soula S., S. Chauzy et S. Despiau, 1987 : Comment on electric field recovery and charge regeneration after lightning discharge, by Pradeep Kumar and J. Rai. Ann. Geophysicae, $5,6,541-544$

Taudière I., 1989 : Analyse du rayonnement électromagnétique VHF-UHF de l'éclair. Application à un modèle d'avancée du précurseur négatif. Thèse de doctorat, soutenue le 12 octobre 1989 à l'université de Paris IV. 